\title{
EFFECTS OF WEAK ANCHORING ON C1 AND C2 CHEVRON STRUCTURES
}

\author{
A. DIAZ, N.J. MOTTRAM and G. McKAY \\ Department of Mathematics, University of Strathclyde, \\ 26 Richmond Street, Glasgow G1 1XH, U.K.
}

We present a theoretical study of the effect of weak anchoring on the transition between $\mathrm{C} 1$ and $\mathrm{C} 2$ chevron structures in smectic C liquid crystals. We employ a continuum theory which allows for variable cone, azimuthal and layer tilt angles. Equilibrium profiles for the director cone and azimuthal angles in the C1 and C2 states are calculated from the standard Euler-Lagrange minimisation of the total energy of the system. By comparing the total energies of the $\mathrm{C} 1$ and $\mathrm{C} 2$ states we can determine the globally stable chevron profile and calculate the critical temperature for the C1-C2 transition, which depends on anchoring strength and pretilt angle variations.

Keywords: surface-stabilised ferroelectric liquid crystals; weak anchoring; C1, C2 structure formation.

\section{INTRODUCTION}

Clark and Lagerwall first demonstrated the surface stabilisation of ferroelectric liquid crystals (SSFLCs) in 1980 [1] and demonstrated that it was possible to suppress the chiral helix by confining a smectic $\mathrm{C}^{*}$ material between two parallel substrates. It was soon realised that an understanding of the chevron structure of the smectic layers [2-4] was crucial to exploiting the display possibilities of SSFLCs. Since then a great deal of research has focused on the development of SSFLCs for display purposes. The chevron structure appears when a sample of smectic A, in a bookshelf configuration, when the liquid crystal is cooled to the smectic $\mathrm{C}$ phase. The layer contraction that occurs during cooling is associated with a layer lengthening and thus, in a constrained system where layer continuity is conserved, layer buckling. This buckling may be degenerate so that there are two possible directions for the layer to orientate, leading to $\mathrm{C} 1$ and $\mathrm{C} 2$ chevrons (see fig. 1). This degeneracy of the layer configuration means that different domains of the cell may be in different chevron states resulting in the formation of zigzag defects between domains [3,4]. However, when the surfaces are treated so that a specified director angle is induced ("pretilt") at the substrate, this degeneracy can be broken. Thus, one of the C1 or C2 states will be preferred leading to the removal of defects, a homogeneous device with better optical characteristics.

We will examine the effect of weak pretilt anchoring at the cell substrates on the formation and stability of $\mathrm{C} 1$ and $\mathrm{C} 2$ chevrons. In order to understand and predict the formation of chevrons, we examine director profiles and layer structures as the surface anchoring strength, substrate pretilt angle and sample temperature are varied. 


\section{GEOMETRY OF THE CELL}

We consider a sample of smectic C liquid crystal constrained by two parallel substrates a distance $2 d$ apart. We define the $z$-axis as the direction perpendicular to the cell substrates, the $x$-axis will be the rubbing direction on the substrate and the $y$-axis completes our orthogonal system of reference, see fig. 1. We will model the lower half of the cell, from $z=0$ to $z=d$, and assume that the upper half is equivalent due to symmetry.

We can describe the smectic $\mathrm{C}$ phase as a layered structure where the unit director $\mathbf{n}$ makes an angle $\theta$, the cone angle, with respect to the layer normal. The director $\mathbf{n}$ is then constrained to lie on the surface of a fictitious cone, with vertex angle $2 \theta$. The twist angle $\phi$ denotes the position of $\mathbf{n}$ on the fictitious cone and the layer tilt angle $\delta$ describes the tilt of the layers from the substrate normal, see fig. 1. Using the appropriate rotation matrices, $\mathbf{n}$ can be described as

$$
\mathbf{n}=\left[\begin{array}{c}
\cos \theta(z) \cos \delta(z)+\sin \theta(z) \sin \delta(z) \cos \phi(z) \\
-\sin \theta(z) \sin \phi(z) \\
\cos \theta(z) \sin \delta(z)-\sin \theta(z) \cos \delta(z) \cos \phi(z)
\end{array}\right]
$$

The $\mathrm{C} 1$ and $\mathrm{C} 2$ chevrons are then characterised by, respectively, a positive or negative layer tilt angle $\delta$ in the lower half of the cell. Here, we have assumed that all angles depend only on the $z$-coordinate throughout the cell.

\section{ENERGY}

The liquid crystal will exhibit an equilibrium structure that attains a minimum state of the total free energy. We will consider a free energy that incorporates elasticity, thermotropic and surface anchoring effects. In the present work the following simplified elastic energy density is adopted,

$$
W_{\text {elastic }}=\frac{K_{\theta}}{2}\left(\frac{d}{d z} \theta(z)\right)^{2}+\frac{K_{\delta}}{2}\left(\frac{d}{d z} \delta(z)\right)^{2}+\frac{K_{\phi}}{2} \theta(z)^{2}\left(\frac{d}{d z} \phi(z)\right)^{2},
$$

where $K_{\theta}, K_{\phi}$ and $K_{\delta}$ are elastic constants. Note that $W_{\text {elastic }}$ in eq. (2) depends only on the squares of the first derivatives of each angle, cross terms have been neglected. Also, the coefficient of the derivative of $\phi$ is proportional to $\theta^{2}$ since (by the definition of $\phi$ and $\theta$ ) when $\theta$ is zero, $\phi$ is undefined in which case gradients in $\phi$ should not contribute to the energy. Even though this is a simplified form of the elastic energy, the following results will be qualitatively the same if a more complicated and more accurate elastic energy [6] is used.

The thermodynamic energy density is derived from a Landau expansion of the true thermotropic energy functional in the order parameter $\theta[5]$,

$$
W_{\text {therm }}=\frac{1}{2} \alpha \Delta T \theta(z)^{2}+\frac{1}{4} b \theta(z)^{4}+\frac{1}{6} c \theta(z)^{6},
$$


where $\Delta T=T-T_{A C}$ is the temperature measured from the smectic A to smectic $\mathrm{C}$ transition temperature $T_{A C}$, and $\alpha, b, c$ are the Landau coefficients. We assume that $\alpha, b$ and $c$ are temperature independent.

In this model we will also assume an empirically derived relationship [7] between the layer tilt and the cone angle, $\delta=\mu \theta$, where $|\mu|=0.85$ is a typical experimentally determined value $[7,8]$. Therefore, we will model $\mathrm{C} 1$ and $\mathrm{C} 2$ chevrons by assuming $\mu=+0.85$ and $\mu=-0.85$ respectively, when $\theta>0$.

\section{BOUNDARY CONDITIONS}

From geometrical considerations and the assumed symmetry of the chevron structure, the director is constrained to lie in the $x y$-plane at the chevron interface, i.e. $\mathbf{n}_{3}=0$ at $z=d$, the centre of the cell. We also assume that the cone angle $\theta(z)$ is smooth at the chevron interface [9] so that, using (1),

$$
\phi(d)=\cos ^{-1}\left(\frac{\tan (\delta(d))}{\tan (\theta(d))}\right), \quad \theta^{\prime}(d)=0 .
$$

At the cell substrate we allow a degree of weak anchoring. This is modelled via a Rapini-Papoular type surface anchoring [10],

$$
W_{\text {surface }}=\frac{1}{2} \tau_{0}\left(1-\left(\mathbf{n} \cdot \mathbf{n}_{0}\right)^{2}\right) \text {, }
$$

where $\tau_{0}$ is the anchoring strength and the preferred director orientation at the substrate is

$$
\mathbf{n}_{0}=\left(\cos \left(\alpha_{0}\right), 0, \sin \left(\alpha_{0}\right)\right)
$$

for a pretilt angle $\alpha_{0}$.

By comparing $\mathbf{n}_{\mathbf{0}}$ and the expression for the director $\mathbf{n}$, we observe that the preferred director angles at the substrate $z=0$ are

$$
\phi=\pi \quad \text { and } \quad \theta=\frac{\alpha_{0}}{(1+\mu)} .
$$

Note that the preferred cone angle $\theta$ is significantly different for $\mathrm{C} 1$ and $\mathrm{C} 2$ chevrons due to the change of sign of $\mu$. Combining eqs (2), (3) and (5) we obtain the total energy per unit area in the $x y$-plane,

$$
E=2\left(\int_{0}^{d}\left(W_{\text {elastic }}+W_{\text {therm }}\right) d z+\left.W_{\text {surface }}\right|_{z=0}\right) .
$$

\section{NUMERICAL CALCULATIONS}

The director configuration will be determined by minimisation of the total energy $E$ of the liquid crystal layer, after non-dimensionalising via $z \rightarrow z / d$. Numerical solutions for the cone and twist angles are obtained from the Euler-Lagrange equations derived 
from the total energy in eq. (8). Some examples of equilibrium profiles are shown in figs 2 and 3. In these and subsequent figures, we have employed the following values taken from the literature [8]: $\alpha=10^{3} \mathrm{Jm}^{-3} \mathrm{~K}^{-1}, b=2.1 \times 10^{6} \mathrm{Jm}^{-3}, c=$ $8.3 \times 10^{6} \mathrm{Jm}^{-3}, d=3 \times 10^{-6} \mathrm{~m}, K_{\phi}=10^{-11} \mathrm{~N}, K_{\delta}=10^{-11} \mathrm{~N}, K_{\theta}=10^{-11} \mathrm{~N}$. All angles shown in subsequent plots are in degrees.

Figures 2 and 3 show the cone angle $\theta(z)$ and azimuthal angle $\phi(z)$ through the cell as pretilt angle and anchoring strength are varied. We see that, in all cases, the cone angle is almost constant in the bulk of the cell at an angle $\theta_{e q}$, the minimum of the thermodynamic energy $W_{\text {therm }}$. This equilibrium cone angle $\theta_{e q}$ depends only on $\Delta T$ and therefore variations of $\tau_{0}$ and $\alpha_{0}$ do not affect the $\theta$ profiles in the bulk. The graphs for $\phi(z)$ (figs 2(b),(d), 3(b),(d)) show a roughly linear twist of the director from the substrate value $\phi=180^{\circ}$ to the chevron interface value. In the $\mathrm{C} 1$ case, $\phi$ at the chevron interface is much lower than in the $\mathrm{C} 2$ case, $150^{\circ}$ in $\mathrm{C} 1$ and $40^{\circ}$ in $\mathrm{C} 2$, due to the change of sign of $\mu$ in the chevron condition in eq. (4).

In figs 2 and 3, any deviation in the cone or twist angle from, respectively, a constant or linear twist profile occurs close to the substrate. In fig. 2, as the pretilt angle is varied the cone angle changes in an attempt to attain the preferred value from eq. (7). In the $\mathrm{C} 1$ case, when $\mu=0.85$, this is approximately half of $\alpha_{0}$ whereas in the $\mathrm{C} 2$ case, when $\mu=-0.85$, this is around seven times $\alpha_{0}$. In fig. 2(c) we see that the anchoring strength is not sufficient to achieve such a high surface cone angle.

The behaviour is different as the anchoring strength varies. In fig. 3 we see that whilst in the $\mathrm{C} 2$ case $\phi$ remains close to $\pi$ at the substrate, in the $\mathrm{C} 1$ case $\phi$ reduces significantly, to the value prescribed by the chevron interface condition. This indicates that, as the anchoring strength is decreased, the anchoring in the C1 case "breaks" before the anchoring in the $\mathrm{C} 2$ case. In fig. $3(\mathrm{~b})$, at $\tau_{0}=10^{-5} \mathrm{Nm}^{-1}$, the azimuthal angle at the surface is far from the preferred substrate value $\left(\phi(0)=180^{\circ}\right)$ but has reduced to the value at the chevron interface $\phi \approx 40^{\circ}$ throughout the cell in order to minimise the elastic energy. However, in fig. $3(\mathrm{~d})$ at $\tau_{0}=10^{-5} \mathrm{Nm}^{-1}$, the azimuthal angle at the surface remains close to the preferred value. This is due to the fact that there exists high azimuthal elastic energy in the C1 state when the director twists through the large azimuthal angle from the preferred substrate angle to the chevron interface angle $\left(180^{\circ} \rightarrow 40^{\circ}\right)$. In the $\mathrm{C} 2$ state the required twist is smaller $\left(180^{\circ} \rightarrow 150^{\circ}\right)$ and thus the elastic energy is smaller.

\section{COMPARISON OF ENERGIES}

We now examine the total energy of the system, $E$, for the $\mathrm{C} 1$ and $\mathrm{C} 2$ states as the temperature, the substrate pretilt and anchoring strength are varied. From fig. 4 we see that the $\mathrm{C} 2$ state has the lower energy for small pretilt angles. As the pretilt angle increases, the $\mathrm{C} 1$ state lowers in energy, with a crossover at a critical pretilt. This behaviour can be explained by considering the preferred value of $\theta$ at the substrate compared to the bulk equilibrium cone angle. If we consider the energy due to the cone angle variations (the thermotropic energy and the cone angle elastic energy) we 
see that the minimum energy state exists when the preferred substrate cone angle value happens to coincide with the thermotropic equilibrium cone angle. (In fact, the $\theta$ dependent energy terms turn out to be the most significant energy term). The minimum energy state is then a constant cone angle $\theta(z)=\theta_{e q}$. If the preferred substrate cone angle differs from $\theta_{e q}$ there will be an associated energy increase. In the $\mathrm{C} 1$ state the preferred value of the cone angle at the substrate is $\alpha_{0} /(1+|\mu|)$, whereas in the $\mathrm{C} 2$ state, when $\mu<0$, the preferred value is $\alpha_{0} /(1-|\mu|)$. For the value $|\mu|=0.85$ the difference between these preferred values in the $\mathrm{C} 1$ and $\mathrm{C} 2$ states is significant $\left(0.54 \times \alpha_{0}\right.$ and $6.7 \times \alpha_{0}$. The chevron state $(\mathrm{C} 1$ or $\mathrm{C} 2)$ which has a preferred value closer to the equilibrium cone angle will be of the lowest energy.

For a fixed temperature the equilibrium cone angle is fixed. For a sufficiently low pretilt value both preferred substrate cone angle values for the $\mathrm{C} 1$ and $\mathrm{C} 2$ states, $\alpha_{0} /(1+|\mu|)$ and $\alpha_{0} /(1-|\mu|)$ will be less than $\theta_{\text {eq }}$ but, since $\alpha_{0} /(1+|\mu|)<\alpha_{0} /(1-|\mu|)$, the $\mathrm{C} 2$ value will be closer to $\theta_{e q}$. We therefore expect the $\mathrm{C} 2$ state to have a lower energy than the C1 state. This expectation is verified in fig. 4 where we see that the $\mathrm{C} 2$ state has lower energy at low pretilt values. As the pretilt value increases the C2 preffered cone angle $\alpha_{0} /(1-|\mu|)$ becomes much greater than $\theta_{e q}$ and thus the $\mathrm{C} 1$ state, where $\alpha_{0} /(1+|\mu|)$ remains close to $\theta_{\text {eq }}$, will be of lower energy.

In fig. 4(a) we see that as $\Delta T$ decreases, and consequently $\theta_{e q}$ increases, the crossover in energy is delayed until higher values of $\alpha_{0}$. This is clear from the above explanation since the point where $\alpha_{0} /(1-|\mu|)=\theta_{e q}$ will now occur at a higher value of $\alpha_{0}$. For weaker anchoring strengths, see fig. 4(b), the director at the substrate has less of a tendency to attain the preferred value and the crossover in energies occurs only when the pretilt is relatively large.

\section{SUMMARY}

We have calculated director and chevron profiles in a smectic $\mathrm{C}$ liquid crystal as a function of anchoring strength, pretilt and temperature. We have found the crossover on energies between the $\mathrm{C} 1$ and $\mathrm{C} 2$ states is highly dependent on both anchoring strength and pretilt value. As anchoring strength is decreased we have demonstrated that the director anchoring breaks in the $\mathrm{C} 1$ state before the $\mathrm{C} 2$ state. We have also shown that with increased pretilt angle and increased anchoring strength the $\mathrm{C} 2$ state is preferred over the $\mathrm{C} 1$ state.

\section{ACKNOWLEDGEMENTS}

NJM and AD wish to thank EPSRC and the University of Strathclyde respectively for financial support.

\section{REFERENCES}

[1] N. A. Clark and S. T. Lagerwall, Appl. Phys. Lett., 36, 899, (1980).

[2] T. P. Rieker, N. A. Clark, G. S. Smith, G. S. Parmar, E. B. Sirota and C. R. 
Safinya, Phys. Rev. Lett., 59, 2658, (1987).

[3] N. A. Clark, T. P. Rieker, Phys. Rev. A, 37, 1053, (1988).

[4] S. T. Lagerwall, Ferroelectric and Anti-ferroelectric Liquid Crystals, Wiley-VCH (1999).

[5] S. A. Pikin, Structural Transformations in Liquid Crystals, Gordon \& Breach, New York (1991).

[6] I. W. Stewart, The Static and Dynamic Continuum Theory of Liquid Crystals, Taylor and Francis, London and New York (2004).

[7] S. J. Elston and J. R. Sambles, The Optics of Thermotropic Liquid Crystals, Taylor and Francis, London and New York (1998).

[8] F. Giesselmann, P. Zugenmaier, I. Dierking, S. T. Lagerwall, B. Stebler, M. Kaspar, V. Hamplova and M. Glogarova, Phys. Rev. E, 60, 598, (1999).

[9] N. J. Mottram and S. J. Elston, Euro. Phys. J. B, 12, 277 (1999).

[10] D. Demus, J. W. Goodby, G. W. Gray, H. W. Spiess, V. Vill, Handbook of Liquid Crystals Vol. 1, Wiley-VCH (1998). 


\section{List of Figures}

1 (a) Director configuration within a tilted layer illustrating the cone angle $\theta$, azimuthal angle $\phi$ and layer tilt angle $\delta$; (b) Layer configuration for $\mathrm{C} 1$ and $\mathrm{C} 2$ chevron structures. . . . . . . . . . . . . 7

2 Director angles $\theta$ and $\phi$ profiles for the C1 and C2 chevron states as pretilt angle $\alpha_{0}$ varies for the parameter values $\tau_{0}=10^{-3} \mathrm{Nm}^{-1}$ and $\Delta T=-1$. (a), (c) cone angle $\theta(z)$ for $\mathrm{C} 1$ and $\mathrm{C} 2$ respectively; (b), (d) azimuthal twist angle $\phi(z)$ for $\mathrm{C} 1$ and $\mathrm{C} 2$ respectively. . . . . . . . 8

3 Director angles $\theta$ and $\phi$ profiles for the $\mathrm{C} 1$ and $\mathrm{C} 2$ chevron states as the anchoring strength $\tau_{0}$ varies for the parameter values $\alpha_{0}=1^{\circ}$, $\Delta T=-1$. (a), (c) cone angle $\theta(z)$ for $\mathrm{C} 1$ and $\mathrm{C} 2$ respectively; (b), (d) azimuthal twist angle $\phi(z)$ for $\mathrm{C} 1$ and $\mathrm{C} 2$ respectively. . . . . . . . . . 9

4 Comparison of energies for $\mathrm{C} 1$ and $\mathrm{C} 2$ chevrons as pretilt varies for: (a) different values of $\Delta T$, with $\tau_{0}=10^{-3} \mathrm{Nm}^{-1}$; and (b) for different values of $\tau_{0}$, with $\Delta T=-5$. Dashed lines indicate the $\mathrm{C} 1$ chevron energy and solid lines indicate the $\mathrm{C} 2$ chevron energy. . . . . . . . . . . 10 


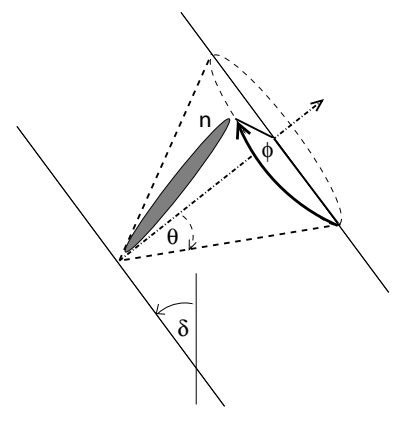

(a)

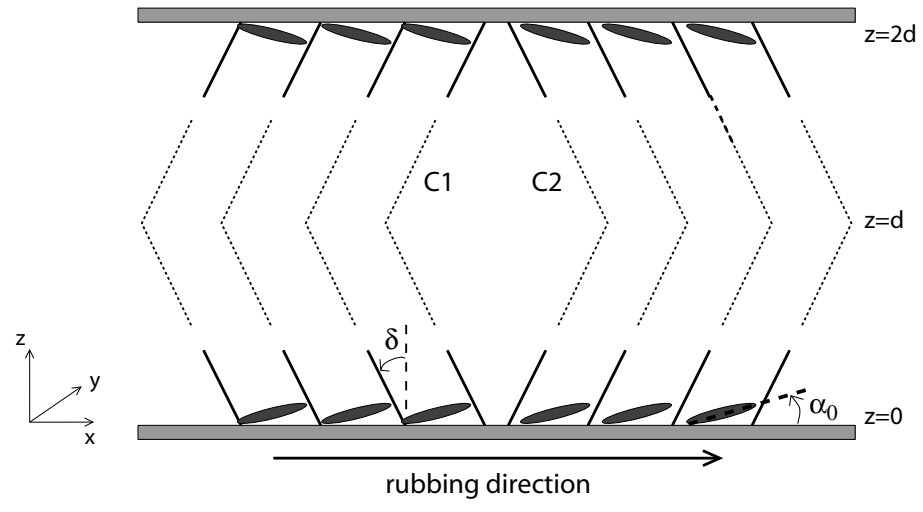

(b)

Figure 1: (a) Director configuration within a tilted layer illustrating the cone angle $\theta$, azimuthal angle $\phi$ and layer tilt angle $\delta$; (b) Layer configuration for C1 and C2 chevron structures. 

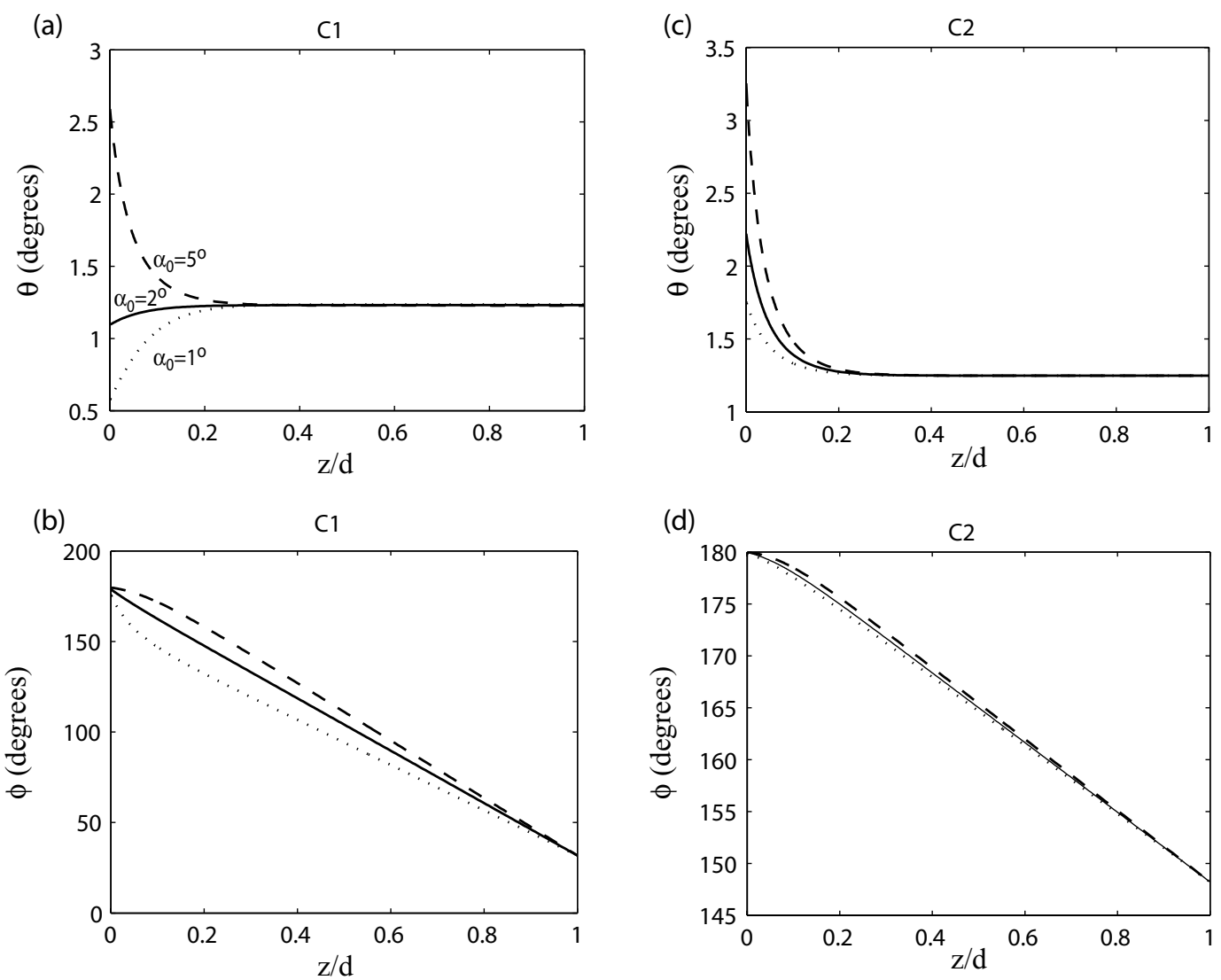

Figure 2: Director angles $\theta$ and $\phi$ profiles for the $\mathrm{C} 1$ and $\mathrm{C} 2$ chevron states as pretilt angle $\alpha_{0}$ varies for the parameter values $\tau_{0}=10^{-3} \mathrm{Nm}^{-1}$ and $\Delta T=-1$. (a), (c) cone angle $\theta(z)$ for $\mathrm{C} 1$ and $\mathrm{C} 2$ respectively; (b), (d) azimuthal twist angle $\phi(z)$ for $\mathrm{C} 1$ and $\mathrm{C} 2$ respectively. 

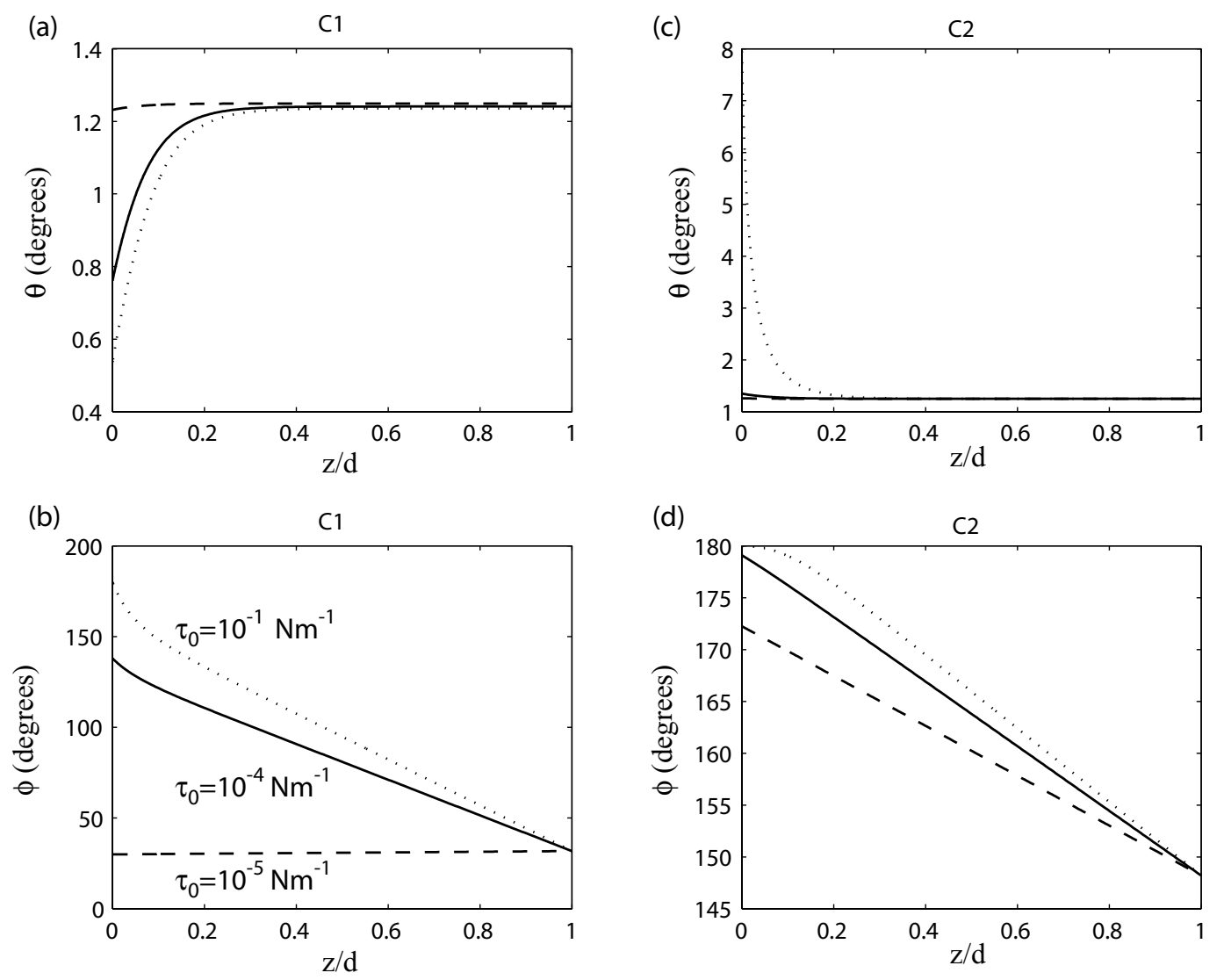

Figure 3: Director angles $\theta$ and $\phi$ profiles for the $\mathrm{C} 1$ and $\mathrm{C} 2$ chevron states as the anchoring strength $\tau_{0}$ varies for the parameter values $\alpha_{0}=1^{\circ}, \Delta T=-1$. (a), (c) cone angle $\theta(z)$ for $\mathrm{C} 1$ and $\mathrm{C} 2$ respectively; (b), (d) azimuthal twist angle $\phi(z)$ for $\mathrm{C} 1$ and $\mathrm{C} 2$ respectively. 
(a)
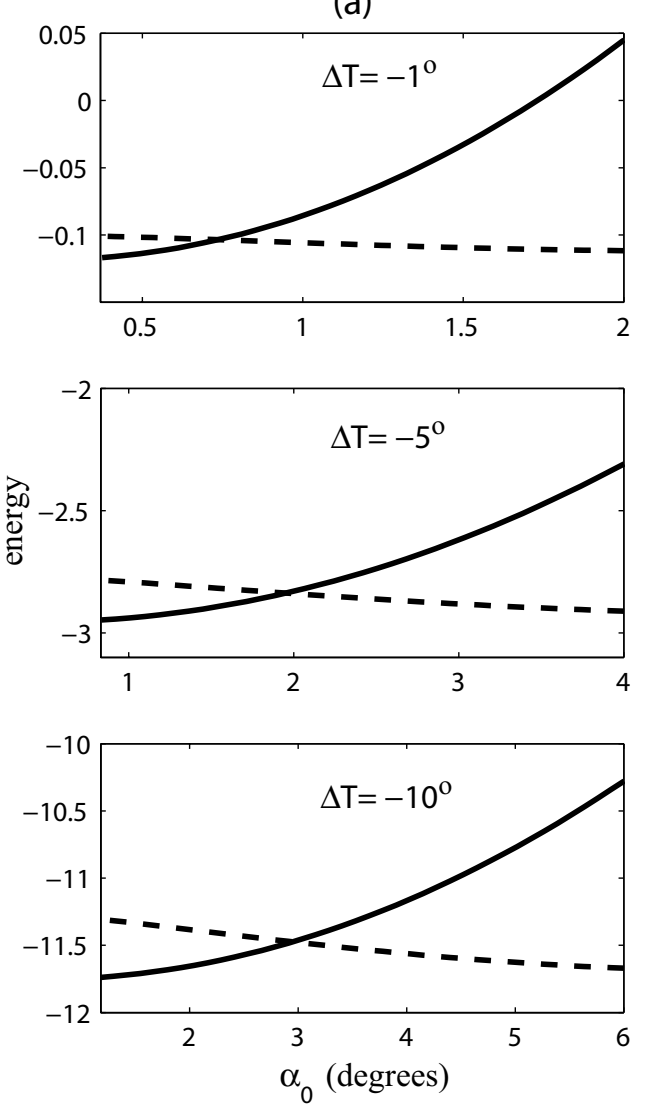

(b)
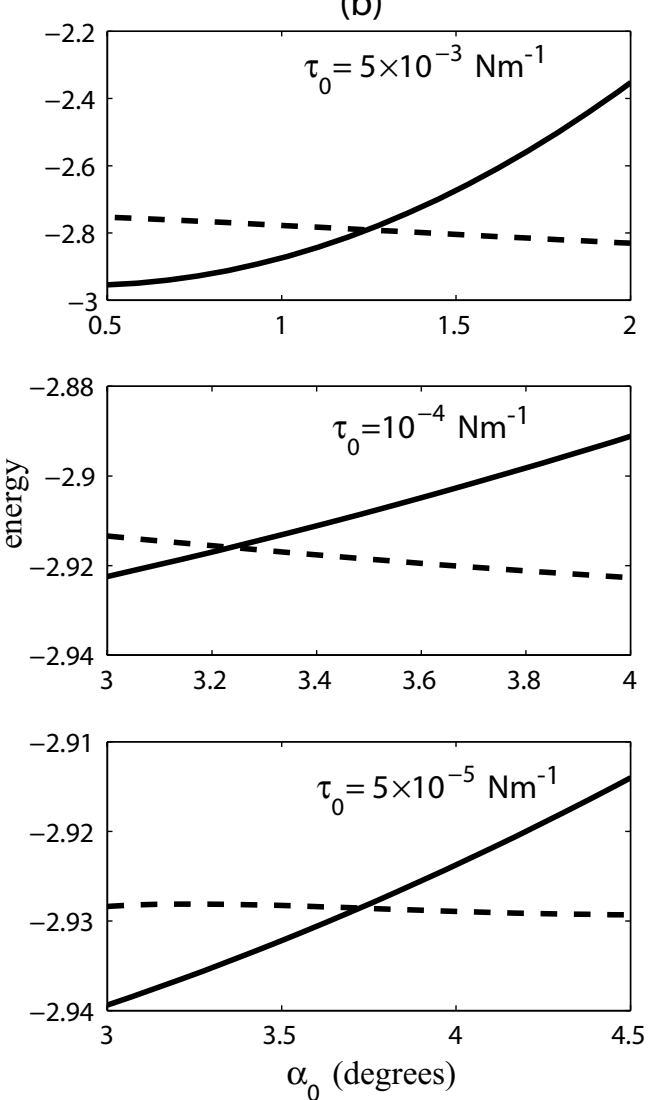

Figure 4: Comparison of energies for $\mathrm{C} 1$ and $\mathrm{C} 2$ chevrons as pretilt varies for: (a) different values of $\Delta T$, with $\tau_{0}=10^{-3} \mathrm{Nm}^{-1}$; and (b) for different values of $\tau_{0}$, with $\Delta T=-5$. Dashed lines indicate the $\mathrm{C} 1$ chevron energy and solid lines indicate the C2 chevron energy. 